\title{
Distributed Geophysical Exploration of Enceladus and Other Ocean Worlds
}

\author{
A White Paper for the NRC Planetary Science and Astrobiology Decadal Survey
}

\section{Authors:}

S. D. Vance1, M. Behounkova2, B. G. Billsı, P. Byrne3, O. Cadek2, J. Castillo-Rogez1, G. Choblet4, K. Hughson5, T. Hurford6, S. Kedarı, James Keane1, Edwin Kite, A. H. Lobo8, A. G. Marusiak1, M. Melwani Daswani1, M. P. Panning1, R. S. Park1, B. E. Schmidt, K. Sladkova2, K. M. Soderlund,, C. Sotin1, O. Soucek11, S. Stähler12, G. Steinbrügge ${ }_{13}$ S. Tharimena1, A. Thompson6, G. Tobie3, W. Song13, and S. Wang 13

1Jet Propulsion Laboratory, California Institute of Technology, (svance@jpl.caltech.edu), 2Charles University, Faculty of Mathematics and Physics, Department of Geophysics, Prague, Czech Republic, 3 North Carolina State University, 4Laboratoire de Planétologie et Géodynamique, Université de Nantes, France, 5 Georgia Institute of Technology, ${ }_{6}$ NASA Goddard Space Flight Center, 7 University of Illinois, Chicago ${ }_{8}$ California Institute of Technology, 9 University of Texas at Austin, Institute for Geophysics, ${ }_{11}$ Mathematical Institute of Charles University, Prague, ${ }_{12}$ Institute for Geophysics, ETH Zürich, Switzerland, ${ }_{13}$ University of Georgia, USA 


\section{Introduction}

Networked multi-node missions should be developed in the coming decade, and should be considered as part of the next ocean worlds mission. Such networks could be key to interpreting the geophysical and geochemical context of any sampled materials in order to understand the context of habitability needed for interpreting any putative signature of life. Here, we describe the benefits of distributed exploration with a focus on Saturn's moon Enceladus, a key target in the search for life.

\section{The geophysical context of habitability at Enceladus}

A future in-situ mission to Enceladus could be the lowest-cost mission to determine if life exists beyond Earth, since material from the subsurface ocean, where the presence of hydrothermal activity has been demonstrated by the Cassini mission, is available on the surface after being ejected by plumes and then settling on the surface. In addition, the low radiation environment of Enceladus would not significantly alter the chemical makeup of samples recently deposited on the surface or lofted into space. Further context on life detection at Enceladus can be found in the companion paper led by Morgan Cable entitled "The Case for a Return to Enceladus". Placing any candidate signatures for life in context requires further knowledge of the interior of Enceladus.

The interior determines the amount of energy available for life. Water-rock interactions can provide the chemical energy and materials for life [e.g., 1-3]. These interactions also determine the ocean's chemistry, including the ionic strength and $\mathrm{pH}$ that constrain the available energy for metabolism [4]. The fluid composition is a tell-tale for the global thermal and tidal evolution. In the case of Enceladus, it has been proposed that water in interstitial pore spaces enables tidal flexing of the rocky core that gives rise to $>10 \mathrm{GW}$ of tidal heat [5]. Tidal heating may facilitate water-rock reactions, and promote element flux at the ocean-rock interface as the primary control of the ocean's composition. For Enceladus, reactions between the ocean and rock may have led to extensive ( $>30 \%)$ carbonation of the rocky core [6]. Direct sampling of plume ejecta and deposits provides information about the ocean's composition and can be used to infer the extent of water-rock interaction but, in contrast to geophysical methods (see below), cannot be used to accurately constrain the extent and volume of the watersaturated zone of the rocky core, where chemical and energetic flux may be taking place.

Ocean circulation controls the conveyance of materials generated by water-rock interactions to the surface. The overall configuration of heating at the ocean bottom and cooling at the ocean-ice interface [5] facilitates thermally driven circulation where convective activity should tend to homogenize the ocean interior $[7,8]$. Mechanical drivers may also contribute to mixing in the ocean interior [e.g., 9,10]. The presence of global icy lithospheres that are in direct contact with the oceans may support salinitydriven circulation [11-13]. Distinct regions of ice melt and freezing could sustain the observed pole-toequator ice thickness gradients $[14,15]$. The introduction of freshwater or brine from phase changes provides potential energy that in a rotationally constrained and stratified environment is likely to be released as turbulent eddies that will impact large-scale heat and tracer transport [16]. Higherresolution estimates of the ice-shell thickness distribution, composition, and dynamics would assist in characterizing the forcings at the ocean-ice interface. Such improved characterization would improve estimates of the ocean structure and circulation, as well as of heat and nutrient transport from the core to the ice. Similarly, further numerical simulations are needed to consider the mechanical and chemical coupling between the interior circulation and the ice shell. 


\section{Evaluating science goals and mission architectures at Enceladus}

The context of sampled materials will be key to understanding any potential biosignatures. Placing collected samples in context requires further knowledge of the interior of Enceladus. For example, a putative biosignature in the form of a mass distribution of hydrocarbons consistent with life on Earth must be interpreted in light of the inferred mineral composition, vigor of water-rock alteration through time, and corresponding inferred fluxes to the ocean of reductants and oxidants that create the energetic chemical conditions for different metabolic reactions.

Key contextual questions addressed by geophysical measurements to assess the astrobiological potential include:

1. How is tidal dissipation distributed between the icy crust, ocean, and rocky core?

2. Why does the North Pole differ from the South Pole?

3. What is the spatial occurrence of ongoing high-temperature water-rock interactions?

4. What is the resurfacing rate?

5. What is the rate of delivery of any oxidants to the interior?

6. How do materials at the ice-ocean interface fractionate due to ice formation and melt processes, and by the workings of the eruptive south polar plumes?

7. How, and to what extent, does the ocean transport materials to the ice-ocean interface and toward the icy surface?

Because the long-term orbit of a spacecraft around Enceladus is likely to be unstable on the timescale of tens of days, it is important to consider landed geophysical elements as supporting or independent assets capable of probing geophysical processes over longer timescales. For a discussion of geophysical exploration focused on orbiting architectures, we refer the reader to the white paper by Ermakov et al. The following sections are intended to serve as guidelines for the further development of analogue studies, technologies, and mission goals in the coming decade.

\section{Characterizing Activity at the South Polar Terrain (SPT)}

High-precision imaging and altimetry from orbit would improve our understanding of the SPT while also enabling geodetic studies, like measuring libration, obliquity, and tidal deformation $\left(h_{2}\right)$, which are sensitive to Enceladus's interior structure. Repeat-pass altimetry with synthetic-aperture radar (InSAR) $(10 \mathrm{~cm}$ resolution) or LiDAR (light detection and ranging; $10 \mathrm{~s}$ of $\mathrm{cm}$ ) would allow interferometric analyses to place bounds on estimates of the horizontal and vertical motions of ice and opening of the major Tiger Stripe fracture sets from which plume jets originate; help detect active fracturing of the shell and provide constraints on deriving the thickness of the icy crust $[17,18]$. Laser altimetry would have the advantage of aiding a gravity investigation and revealing long-wavelength topography on a global scale. Mapping topographic features and thermal output with greater spatial coverage and resolution than Cassini's investigations would facilitate understanding of the heat distribution and active features in the ice, as well as helping to improve our understanding of the tectonic and geological evolution of the SPT itself, and its role in sustaining of the south polar plumes.

Electromagnetic (EM) methods are optimal for deducing both chemical properties and structure. EM can infer vertical structures that cannot be directly imaged via radar. Both passive (magnetotelluric, MT) and active (transient electromagnetic, TEM) methods are well-suited for detecting fluid pathways linking Enceladus's surface to the subsurface ocean. Regions of conducting liquids and relatively resistive ice can be detected and distinguished by their corresponding resistivity signatures detectable by the strength of inductive currents produced by either active loops (TEM) or from naturally occurring sources (MT). Magnetotelluric sounding at Enceladus would use time-varying natural fields that induce secondary fields in the subsurface. MT takes advantage of the "skin-depth effect" by measuring the ratio of the orthogonal horizontal components of the local electric and magnetic fields at 
a range of frequencies, which is used to reconstruct the vertical resistivity structure of the subsurface below the station, and requires little knowledge of the structure of the primary field or its natural variation [19].

Landed single-station MT measurements can provide insights into the subsurface structure of Enceladus' ice shell. The sensitivity to lateral resistivity variations becomes a strength of the technique if multiple nodes are utilized (Figure 1). Where resistivity variations are likely dominated at the south pole by the Tiger Stripes [20], even a two-station MT experiment in the SPT separated by a few kilometers could bound the resistivity and hydrological structure of the plume jets plumbing system. An advantage of the TEM method is that the details of the source field are known exactly, but this requires high power [22]. Conditions at Enceladus are sufficient for MT measurements with reduced power consumption. TRL 6 MT prototypes for Europa exist [22] that require little modification for Enceladus, and could leverage existing heritage of other flown EM systems.
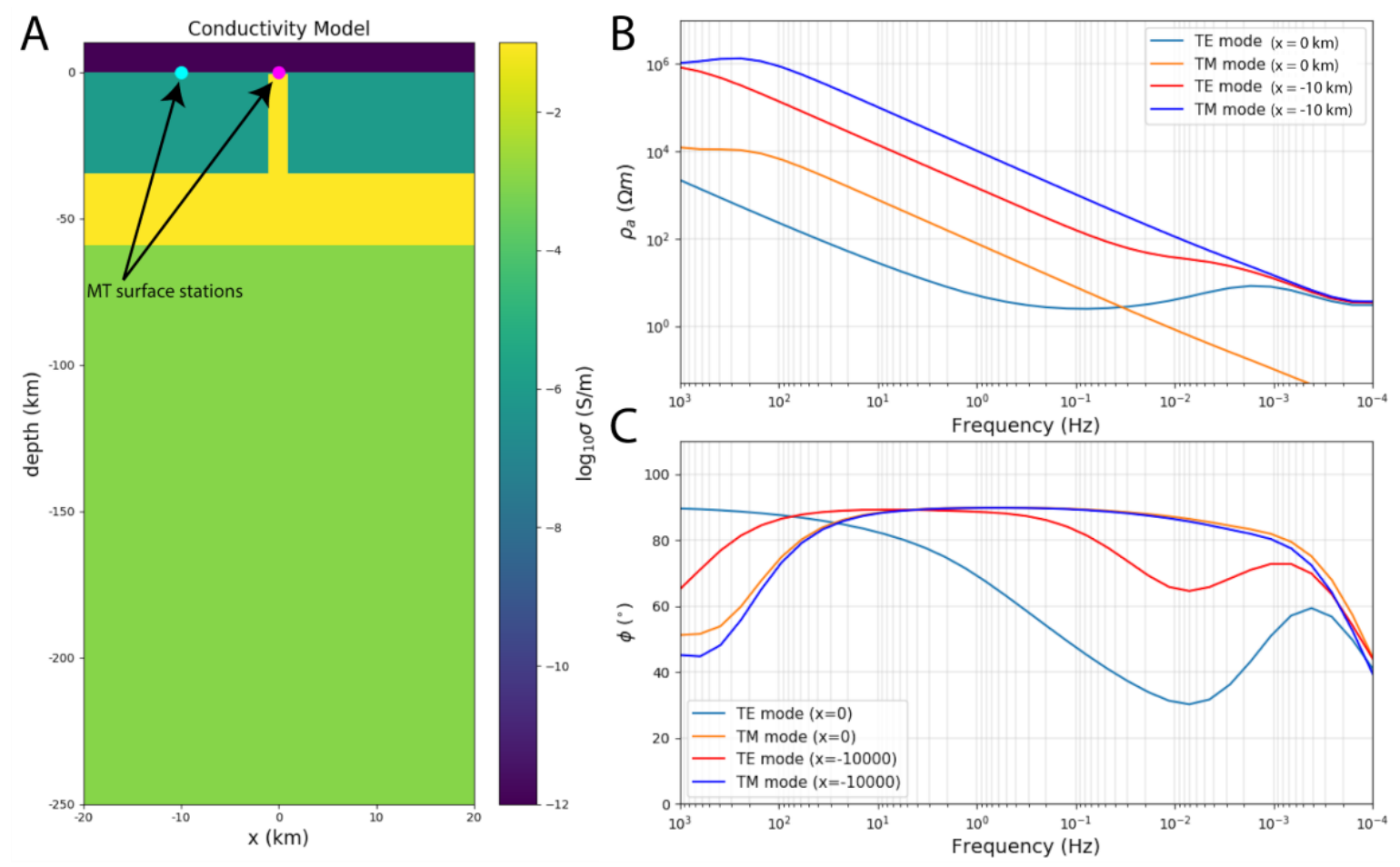

Figure 1. Example two-station MT survey near a conductive, fluid-filled fracture. (A) The conductivity model includes a fracture through the ice-shell (dark green) filled from the ocean (yellow) above a silicate interior (light green). (B) The apparent resistivity and (C) phase angle of the complex impedance as measured by the two MT stations. In this instance, the difference in the behavior of the TE and TM mode at each station in the phase angle illustrates the departure from a uniformly layered subsurface, whereas the differences between the modes and stations in apparent resistivity helps contextualize the magnitude and nature of this departure. 


\section{Assessing global structure}

Global topography, libration, and the static low-degree gravity potential and its time variations can be used to assess ice shell thickness, thickness variations, and mechanical properties of the rocky core and ice shell (white paper: Ermakov et al.). Numerical models (Figure 2) illustrate how a deformable core's contribution to such variations can be discerned if the structure and composition of the interior can be probed independently through other techniques such as seismology. Although gravity measurements might be best conducted from orbit, a landed experiment would provide complementary data, and would be best suited for measuring surface tilt.
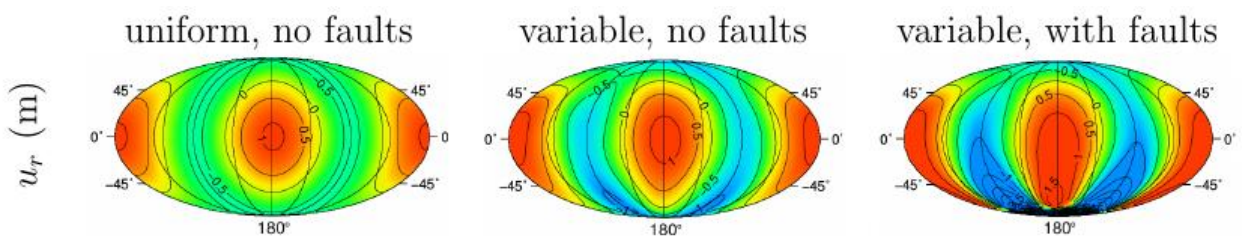

radial displacement, surface, sub-Saturnian point
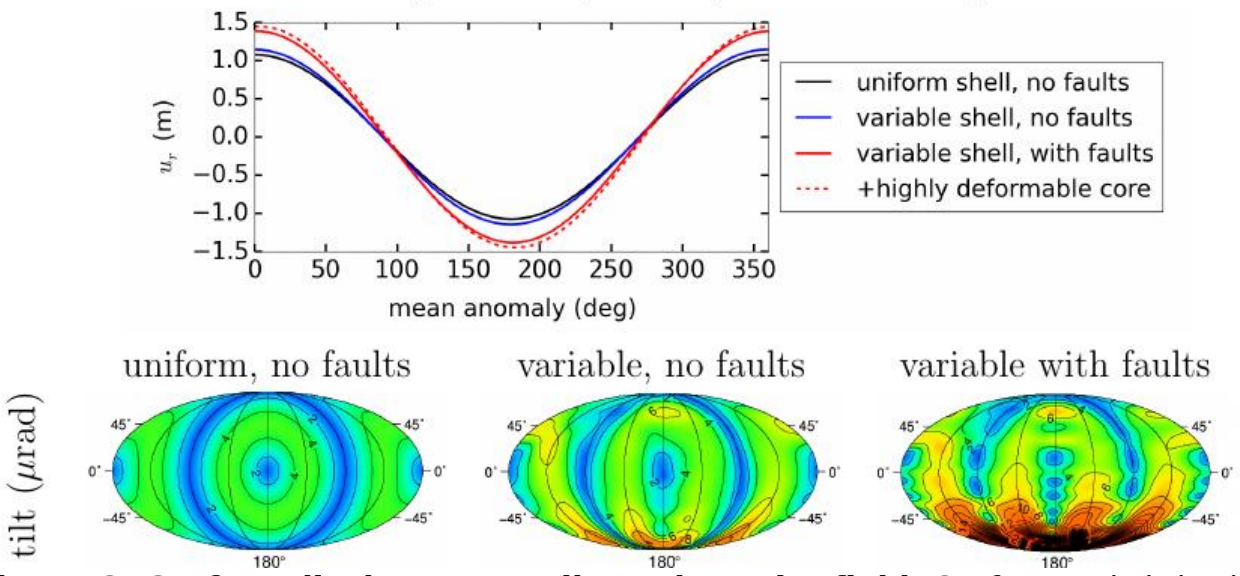

Figure 2. Surface displacement, tilt, and gravity field. Surface radial displacement $u r$ and tilt are for models with uniform or variable ice shell thickness (average thickness $20 \mathrm{~km}$ ) and with or without faults. Gravity anomalies $\Delta g$ at the surface for the model with and without deformable core, variable ice shell thickness with faults. Results based on [21] for elastic ice shell and highly deformable and dissipative ( $>50 \mathrm{GW})$ parameterized core. All maps are shown at periapsis.

Surface displacement on the tidal time scale of 1.37 days is most sensitive to deformation of the ice shell, whereas the effect of the core deformation on the surface displacement is limited $(<10 \%)$. Globally, the radial displacement on the tidal time-scale is represented by deformation tidal Love number $\mathrm{h}_{2}=\frac{g u_{r}}{V}$ and is mostly controlled by ice shell thickness and the shell's rigidity. Based on numerical experiments, the range between 0.17-0.03 (corresponding to radial displacement up to a few meters, Figure 2) are expected for the thickness of the ice shell between 5 and $30 \mathrm{~km}$. The displacement is, however, affected by the local ice shell thickness [e.g., 15,23-25]. Resonant inertial waves in the ocean may also amplify the tidal response [26], making the tidal signal more complex. However, the mechanical coupling between the ocean and the ice shell with variable thickness still needs to be modeled. A possible local increase in radial deformation (up to $8 \mathrm{~m}$ ) in the vicinity of the south pole can be also attributed to the presence of the faults there [20,25; Figure 2]. These mostly local effects can also influence the value of the Love numbers. In the case of a model with an averaged $20 \mathrm{~km}$ thick shell, $h_{2}$ increases from 0.048 to 0.080 if variable ice shell thickness and faults are taken into account. Due to breaking of the radial symmetry and consequent spectral leakage, the Love number splitting and apparent phase advancement of the deformation (up to $20^{\circ}$ for $h_{20}$ ) of these models are predicted. To distinguish between possible ice shell structure and faults, the measurements would require a precision of $h_{2}$ higher than 0.01 , corresponding to a radial displacement of $30 \mathrm{~cm}$. The lateral displacement is $\sim 2$ $x$ smaller, corresponding to a lateral displacement of $15 \mathrm{~cm}$. Various techniques may be considered to measure these displacements. 
Altimetry will be more sensitive to radial displacements, while optical imaging will provide more accurate determination of the lateral displacements. Optical imaging will also allow a precise determination of the shell libration, which is an essential prerequisite to detect subtle lateral displacements from faulting because of tidal deformation. InSAR techniques, as well as multiple beacons at the surface, may be alternative/complementary techniques. For a lander system, the surface deformation would also be measurable in the surface tilt. The tilt at the equator is predicted to vary by up to $4 \mu \mathrm{rad}$. Regionally, it can reach several 10 s of $\mu$ rad if the shell is locally thin or if faults are present (Figure 2). The surface displacement is predicted to be almost independent of the viscoelastic behavior if the ice shell is in the conductive regime [20], and would be mostly sensitive to the local ice shell thickness and potentially to coupling with the ocean dynamics. Measurements of the time-varying gravity field from tides can reveal core and shell deformation signatures. Based on numerical simulations using the approach of [25], the potential Love number $k 2$ with the undeformable core can reach $0.01-0.06$, again depending on the ice shell thickness $(5-30 \mathrm{~km})$, its spatial variations, and the dynamical response of the SPT faults. To distinguish between candidate models, accuracy better than 0.01 would be required. The most sensitive measurement of gravity anomalies would be from the surface due to variations in the gravity field combined with lander displacement. Gravity anomalies from tides can reach several $10 \mathrm{~s}$ of $\mu \mathrm{Gal}$, and can be amplified by regional conditions (Figure 2 ). In the best-case scenario for a highly deformable and dissipative core (Figure 2), the gravity anomaly can exceed 100 $\mu \mathrm{Gal}$ at the surface near the equator. The combined phase lag of the core signal and nearly elastic response of the ice shell might be inferred from gravity and supporting data. Measuring the phase lag of $h_{2}$ and $k_{2}$ simultaneously can further estimate the dissipation in the silicate shell of Enceladus [27].

Detecting the phase lag would be challenging from orbit, but beacon(s) deployed at the surface, especially in the equatorial region, may help this technique reach the required accuracy. More detailed modeling is needed to assess this possibility. The gravity signal may also be detectable by a surface seismometer. The InSight SP, a compact broadband seismometer which could be a candidate for ocean world missions, has self-noise below the $100 \mu \mathrm{Gal}$ level at Enceladus tidal frequency, but likely would also have larger signals due to temperature sensitivity at a similar period due to diurnal temperature variation. Further modeling is needed to determine if the gravity signal can be accurately extracted from a realistic seismic record.

Seismology is the best technique for resolving the deep interior structure of Enceladus, and would be highly complementary to the other techniques described here. A seismic investigation needs to optimize the trade space between the number of seismic nodes, the sensitivity of the seismometer, and the mission duration needed to characterize the thickness and dynamics of the ice and any fluid layers therein. This trade space further includes the thickness, heterogeneity, and dynamics of the ocean, as well as the nature of the underlying rocky interior [28-30]. The small size of Enceladus means that a single seismometer has a strong likelihood of detecting signals originating anywhere at the surface or the interior, while the significant observed plume activity likely indicates ample seismicity for passive seismic techniques.
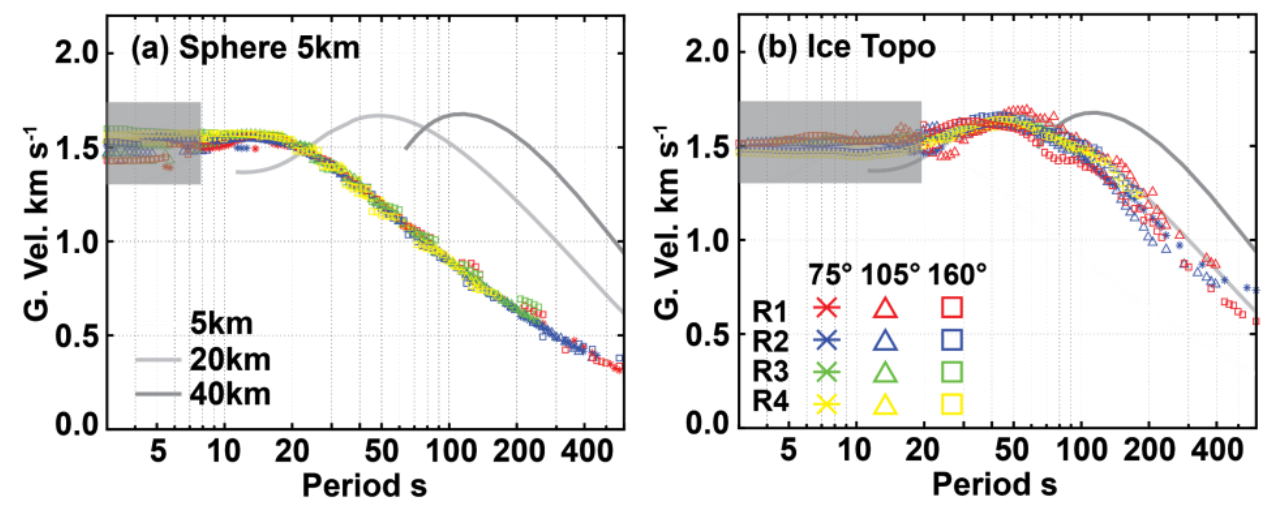

Figure 3: Panels (a, b) show reference $1 \mathrm{D}$ dispersion curves for Enceladus with a 5, 20 , and $40 \mathrm{~km}$-thick ice shell. Symbols show selected fundamental mode surface waves with colors corresponding to their orbit around Enceladus.

Analogue work on Earth has demonstrated the ability of seismic arrays and single stations to determine the structure of the ice and the influences of the local environment (e.g., temperature and tides) on ice [31]. Geophysical analog site studies for ocean worlds have recently assessed the capabilities of single-station 
seismometers and small-aperture arrays to meet key science objectives such as constraining ice shell thickness and locating and identifying sources of seismicity $[32,33]$.

Some seismic waves also will be trapped within the ice shell because it sits on a global ocean with low seismic velocity and no shear waves. These trapped waves can be used to determine the ice thickness at different distances from the seismic station. To illustrate such measurements, seismic waves were propagated through a realistic 3D model of Enceladus using a spectral element wave propagation solver (Figure 3). For a single seismic station located in the model at $75^{\circ}, 105^{\circ}$ and $160^{\circ}$ from the source, the average ice shell thickness variation can be estimated using frequency-time analysis (FTAN). For wavelengths less than the ice shell thickness, surface waves behave similar to Rayleigh waves, and for wavelengths greater than the ice shell thickness, Rayleigh waves transition to full layer flexural waves with an intrinsic dispersion $C_{f l} \alpha \sqrt{f}$ that is independent of the velocity gradient in the ice. The peak associated with this transition is a diagnostic of the ice shell thickness as illustrated in Figure 3, where the average ice-shell thickness of $22 \mathrm{~km}$ was estimated along the path of wave propagation, with (3a) and without (3b) the effects of the ice topography. Another possible diagnostic for ice shell thickness is the Crary wave, which is a harmonic wave trapped within the ice shell [30]. For ice shells less than 40 $\mathrm{km}$ thick, these waves produce resonances above $0.1 \mathrm{~Hz}$ measurable by space-ready seismometers. Due to curvature and variable ice shell, these waves are likely detectable only within $250 \mathrm{~km}\left(50^{\circ}\right)$ of the source.

A single seismic station offers additional tools to infer the interior structure of Enceladus. Body wave reverberations (e.g., SeSn and SeP) can be used to determine the depth of the ocean, and refracted body waves travelling through the rocky interior can be used to infer its properties, such as seismic velocity and density.

The seismic noise environment of Enceladus needs further forward modeling analysis. In contrast to Mars (and likely Venus), where atmospheric effects control the noise of a seismic installation [34-36], tidal cracking in the ice probably controls the seismic noise floor at least between 0.1 and $1 \mathrm{~Hz}$, a powerful band for measuring global quakes [30]. On Europa, expected noise levels range from 10-12 to $10-8 \mathrm{~m} / \mathrm{s} 2 / \mathrm{rtHz}$, depending on the assumed efficiency of converting tidal energy to seismic energy, but expected signals from the largest events would likely be 1-3 orders of magnitude larger. Expected tidal dissipation energy on Enceladus is roughly three orders of magnitude lower [37], but given that trapped waves in the ice shell spread in 2D across the surface of the body, average geometric spreading losses, which scale by surface area, should be smaller on Enceladus thus increasing the expected amplitude of a given ice-cracking event by more than an order of magnitude. Taken together, tidal cracking noise is likely 1-2 orders of magnitude lower on Enceladus than Europa. On Earth, ocean noise dominates seismic noise, but this is dominated by the pressure effects due to interfering ocean surface waves [38]. This may be an important effect on Enceladus due to a subsurface ocean. Recent modeling of turbulent flows at Enceladus should allow for preliminary analysis of the ocean's contribution to seismic noise at the surface.

Magnetic induction measurements may complement gravity and seismic investigations by providing unique constraints on our knowledge of the ocean's conductivity and thickness. In contrast with Jupiter, no tilt of Saturn's intrinsic magnetic field with respect to its spin axis has been observed [17]. Nevertheless, it may be possible to infer the ocean's properties from externally excited oscillations of the magnetic field [18], or using active techniques as described above. This spin-magnetic axis alignment may also be used to search for magnetic induction signals generated by the flow of salty water within the oceans, providing a means to constrain ocean dynamics [39]. 


\section{Networked Architectures}

A smart sensor network can be designed to image the subsurface structure and monitor subsurface events/changes, where the nodes self-form a wireless mesh network and self-perform in-situ computing of subsurface images, enabling real-time continuous monitoring while avoiding costly raw data collection [40]. This approach differs from the traditional data logger approach, and may be essential for a space mission to meet bandwidth constraints for relaying data back to Earth. Such an approach would also increase the resilience of data collection, for example in reducing risk of data loss in the event of a node failure.

We used model ambient noise seismic imaging computations, combined with realistic properties of Enceladus [20], to assess the resolution of seismic networks with 169 to 16 nodes. The denser array offers a high-resolution subsurface image, while the sparser array can still reveal subsurface structure. Comparing the approach of distributed in-situ computing to the traditional telemetry data collection, the data reduction ratio for the satellite communication link is 2700:1.

\section{Conclusion}

A combination of the above methodologies would advance our understanding of the workings and possible habitancy of Enceladus by revealing its structure, composition, and overturning processes. The mission design and instrument suite for the Europa Clipper concept provide mapping coverage and geophysical fidelity generally exceeding those of Cassini [41]. A subset of comparable instruments could be deployed at Enceladus [42]. Concepts for landed missions on icy moons demonstrate the plausibility of safely landing an autonomous laboratory for biosignature characterization and geophysical investigation of habitability $[43,44]$. A networked implementation could offer a leap in the capability to understand the processes supporting habitability, but require support from NASA to conduct further modeling and technical implementation.

\section{References}

[1] Russell, M.J., et al. (2014) Astrobiology, 14, 308-343. [2] Russell, M.J., et al. (2017) Astrobiology, 17, 1265-1273. [3] Barge, L.M. and White, L. M. (2017) Astrobiology, 17, 820-833. [4] Glein, C. et al. (2019) Enceladus and the Icy Moons of Saturn, 39-56. [5] Choblet, G. et al. (2017) Nature Astronomy, 1, 841-847. [6] Glein, C.R., and Waite, J.H. (2020) GRL, 47, e2019GL085885. [7] Soderlund, K.M. et al. (2014) Nat. Geosci. 7 16-19. [8] Soderlund, K. M. (2019) GRL, 46, 87008710. [9] Lemasquerier, D., et al. (2017) JGR:Planets, 122, 1926-1950. [10] Tyler, R. H. (2008) Nature, 456, $770-773$. [11] Lobo, A., et al. (2020) arXiv:2007.06173. [12] Ashkenazy, Y. and Tziperman, E. (2020) arXiv:2006.02242. [13] Zhu, P. et al. (2017). GRL, 44, 5969-5977. [14] McKinnon, W.B. (2015) GRL, 42, 2137-2143. [15] Čadek, O. et al. (2019) Icarus, 319, 476-484. [16] Jansen, M.F. and Nadeau, L.P. (2016) J. Phys. Ocean. 46, 3455-3470. [17] Sandwell D. et al. (2004) JGR, 109, E11003. [18] Rignot E. et al. (2011) Science, 333, 1427-1430. [19] Grimm, R.E. (2002) GRL, 107, 1-29. [20] Yin, A and Pappalardo, R.T. (2015) Icarus, 260, 409-439. [21] Efferso et al. (2019) SkyTEM312 HP. [22] Grimm, R.E. et al. (2020) LPI Proceedings, 1568. [23] Souček, O. et al. (2019) Icarus, 328, 218-231. [24] Beuthe, M. (2016) Icarus, 280, 278-299. [25] Hemingway, D.J. and Mittal, T. (2019) Icarus, 332, 111-131. [24] Cadek, O. et al. (2016) GRL, 43, 56535660. [25] Bĕhounková M. et al. (2018) Astrobiology, 17, 941-954. [26] Rovira-Navarro, M., et al. (2020). Icarus, 343:113711. [27] Hussmann et al. (2016), Celestial Mechanics, 126, 131-144. [28] Vance S. et al. (2018) Astrobiology, 18, 37-53. [29] Vance S. et al. (2018) JGR-Planets, 123, 180-205. [30] Panning P. et al. (2018) JGR-Planets, 123, 163179. [31] Stähler S. et al. (2018) JGR-Planets, 123, 1-27. [32] Podolskiy, E.A. and Walter, F. (2016) Rev. Geophys., 54. [33] Marusiak, A.G. et al. (2019). AGU Fall Proceedings. [34] Marusiak, A.G. et al. (2020). Seism. Res. Lett., 91, 19011914. [35] Lorenz, R.D. and Panning, M. (2018) Icarus, 303, 273-279. [36] Panning, M.P. (2020) JGR-Planets, 125, e2019JE006353. [37] Hurford et al. (2020) Icarus, 338, 113466. [38] Longuet-Higgins (1952) J. Mar. Res. 11, $245-266$. [39] Vance, S. et al. (2020) arXiv:2002.01636. [40] Song, W. et al. (2019) Sensors, 19, 301. [41] Buffington B. et al. (2017) Proc Int Astronautical Congress IAC-17-C1.7.8. [42] Lunine, J.I. et al. (2015) LPSC 1525. [43] Konstantinidis, K. et al. (2015) Acta Astronautica, 106, 63-89. [44] Hand, K. et al. (2017) JPL D-97667. 